\title{
Prognostic significance of PD-LI expression in patients with gastric cancer in East Asia: a meta-analysis
}

This article was published in the following Dove Press journal:

OncoTargets and Therapy

4 May 2016

Number of times this article has been viewed

\author{
Yong-Xuan Liu \\ Xin-Shuai Wang \\ Yu-Feng Wang \\ Xiao-Chen $\mathrm{Hu}$ \\ Jun-Qiang Yan \\ Ya-Li Zhang \\ Wei Wang \\ Rui-Jie Yang \\ Ying-Ying Feng \\ She-Gan Gao \\ Xiao-Shan Feng
}

Department of Oncology, Cancer Institute, The First Affiliated Hospital of Henan University of Science and Technology, Luoyang, People's Republic of China
Correspondence: Xiao-Shan Feng; She-Gan Gao

Department of Oncology, Cancer Institute, The First Affiliated Hospital of Henan University of Science and Technology, No 24, Jinghua Road, Luoyang, 47I000, People's Republic of China

Tel +86 379 I5I 387552 I8

Email samfeng137@hotmail.com; gsgl12258@163.com

\begin{abstract}
The overexpression of programmed cell death-ligand 1(PD-L1) has been observed in gastric cancer (GC). However, whether the expression of PD-L1 in tumor cells or blood serum is associated with the prognosis of patients with GC remains unclear. Therefore, we performed a meta-analysis to evaluate the prognostic significance of PD-L1 expression in GC. Electronic databases were searched systematically. Studies that met the inclusion criteria were included in the meta-analysis. Data concerning the hazard ratio (HR) for overall survival and disease-free survival with a $95 \%$ confidence interval (CI) according to the expression status of PD-L1 evaluated by immunohistochemistry or enzyme-linked immunosorbent assay were extracted. The data were analyzed using a random effects model. Subgroup analyses were proposed. Our results showed that eight studies with 950 patients met the inclusion criteria and were included in the meta-analysis. The pooled HR for overall survival indicated that patients with PD-L1-positive expression had significantly shorter survival time compared with the PD-L1-negative group (HR 1.60, 95\% CI 1.09-2.36, $P=0.012$ ). The pooled HR for disease-free survival demonstrated that the difference between the two groups was not statistically significant (HR 1.02, 95\% CI $0.32-3.20, P=0.98$ ). In conclusion, our results indicate that the evaluation of PD-L1 overexpression in GC tissue or blood serum may be useful in the future as a novel prognostic factor.
\end{abstract} Keywords: PD-L1, gastric cancer, prognostic, meta-analysis, OS, immunohistochemistry

\section{Introduction}

Gastric cancer (GC) is ranked fifth and second in terms of incidence and mortality, respectively, among cancers worldwide. In general, the incidence rates are highest in Eastern Asia, particularly in Korea, Mongolia, Japan, and the People's Republic of China. ${ }^{1,2}$ Although significant changes in multidisciplinary therapies have improved treatment outcomes, the overall prognosis for patients with GC remains poor, especially in advanced stages. ${ }^{3}$

The hallmark of evading immune surveillance is one of the biological capabilities acquired during cancer development. An improved understanding of the molecular mechanisms that govern the host response to tumors has led to the identification of checkpoint signaling pathways that limit the anticancer immune response. ${ }^{4}$ Despite the complexity of cancer immunoediting, ${ }^{5}$ growing evidence suggests that co-inhibitory receptors, such as cytotoxic T lymphocyte-associated antigen-4 and programmed cell death-ligand 1 (PD-L1), play a crucial role in cancer immunoediting, especially in the equilibrium and escape stages. ${ }^{6}$ Recently, immunotherapeutic agents targeting immunosuppressive proteins, such as cytotoxic T lymphocyte-associated antigen-4, PD-L1, and killer cell immunoglobulin-like receptor, have been investigated as potential treatments 
for cancer. ${ }^{7}$ Among these immunotherapeutic agents, an anti-PD-L1 antibody has shown promising clinical efficacy against melanoma and several other cancer types. ${ }^{8,9}$

However, whether the expression of PD-L1 on tumor cells is associated with the prognosis of GC remains unclear. Several recent reports have demonstrated that PD-L1 expression on tumor cells was correlated with poor prognosis; ${ }^{10-15}$ however, not all reports agree with this phenomenon. ${ }^{16,17}$ Therefore, we performed a meta-analysis of data by incorporating all available evidence to evaluate the overall survival (OS) and disease-free survival (DFS) according to levels of PD-L1 in patients with GC with the goal of shedding more light on the development of PD-L1 immune checkpoint-targeted therapy and prognostic prediction in patients with GC.

\section{Materials and methods}

\section{Search strategy}

Two investigators (Y-XL and X-SW) independently performed a systematic search from inception to February 2016 in PubMed, Embase, the Cochrane Library database, Web of Science, China Biomedical Literature Database, and China National Knowledge Infrastructure. The searches were limited to human studies published in either English or Chinese. The keywords were PD-L1, CD274, B7-H1, programmed cell death ligand 1, stomach neoplasms, GC, gastric neoplasm, and stomach cancer.

\section{Inclusion and exclusion criteria}

Eligible studies had to meet the following inclusion criteria: 1) all reported the prognostic value of PD-L1 status for survival in patients with $\mathrm{GC}$; 2) the expression level of PD-L1 was tested by immunohistochemistry (IHC) staining or enzyme-linked immunosorbent assay on the tumor tissue specimens and blood serum; 3) the primary outcome data or survival curves were available. Studies that failed to meet the inclusion criteria were excluded.

\section{Outcomes measures, data extraction, and quality assessment}

Two investigators (Y-XL and X-SW) extracted the data independently, using a prepared information form. Any disagreement was discussed and resolved by consensus in a meeting with a third investigator. The following data were extracted from the included studies: authors, publication year, number of patients, sample source, evaluation method, cutoff value for PD-L1 positivity, primary antibody, and patient survival results of OS and DFS. If data from any of the earlier categories were not reported in the primary study, items were treated as "not available". We did not contact the authors of the primary study to request the information. All the analyses were based on previously published studies, thus no ethical approval or patient consent was required.

\section{Statistical analysis}

Meta-analysis was performed by Cochrane RevMan 5.3.0 (the Cochrane Collaboration, Copenhagen, Denmark). The hazard ratios (HRs) for OS with 95\% confidence intervals (CIs) according to the expression status of PD-L1 were pooled. For the quantitative aggregation of the survival results, HRs and their $95 \%$ CIs were combined to give the effective value, or to estimate the HR from the available data or Kaplan-Meier curves using the methods reported by Tierney et al. ${ }^{18}$ Statistical heterogeneity was assessed using the Cochran's $Q$ and $I^{2}$ statistics. Heterogeneity was considered statistically significant when the $P$-value was $<0.05$ or $I^{2}$ was $>50 \%$. If significant heterogeneity existed, the data were analyzed using a random effects model. ${ }^{19}$ A funnel plot was used to assess the potential publication bias.

\section{Results}

\section{Study selection and characteristics}

A total of 279 articles related to the analysis were identified initially from the literature and subjected to the selection process (Figure 1). Through reading titles, 271 of those were excluded due to nonrelated and duplicate studies. Then, we carefully read the full text of the remaining studies and only eight studies with 950 patients met the inclusion criteria and were included in the meta-analysis. ${ }^{10-17}$

Table 1 summarizes the characteristics of the studies involved in the meta-analysis. Based on an Asian population,

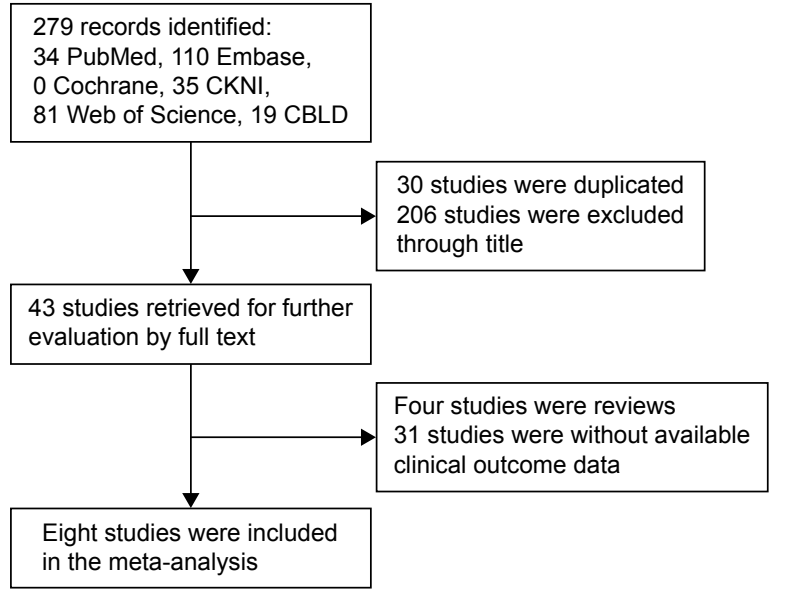

Figure I Flow diagram of literature search and study selection. Abbreviations: CBLD, China Biomedical Literature Database; CKNI, China National Knowledge Infrastructure. 


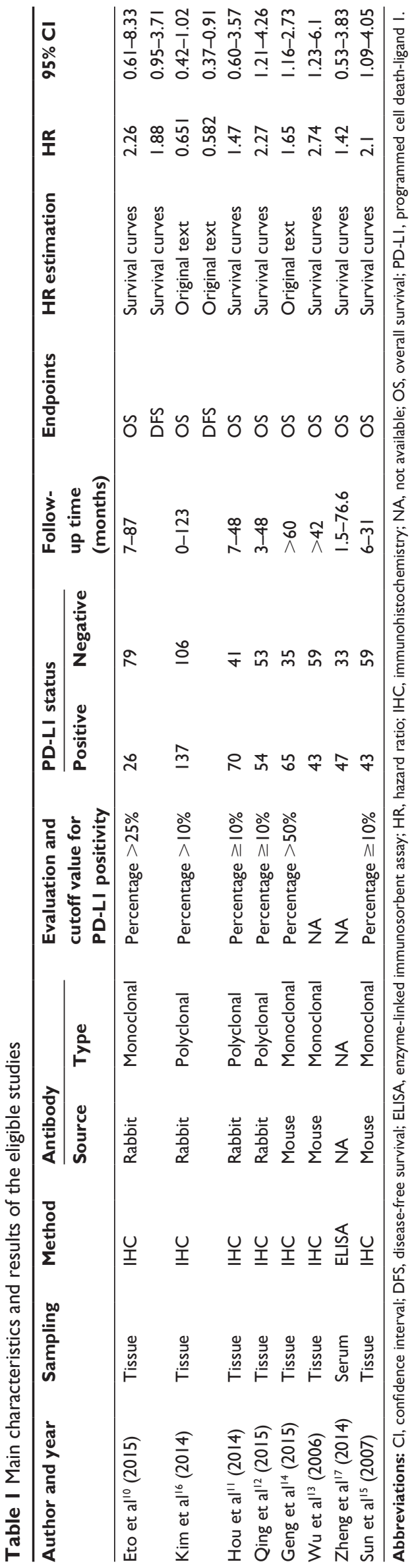

the eight studies eventually included five from People's Republic of China, ${ }^{11-13,15,17}$ two from Japan, ${ }^{10,14}$ and one from Korea ${ }^{16}$ A primary anti-PD-L1 antibody raised in mouse was used in three studies, and four studies used an antibody raised in rabbit. Three studies used a polyclonal antibody, and four studies used a monoclonal antibody. Only one study did not report the clone of the PD-L1 antibody. The cutoff value for PD-L1 overexpression depended on the basis of the percentage of stained cells and the method used.

\section{Meta-analysis results}

A total of eight studies reported the outcome of OS, and 950 patients were included in the analysis. ${ }^{10-17}$ The pooled HR for OS indicated that patients with PD-L1-positive expression had significantly shorter survival time compared with the PDL1-negative group (HR 1.60, 95\% CI 1.09-2.36, $P=0.012$; Figure 2). Only two studies reported the outcome of DFS. ${ }^{10,16}$ The pooled HR for DFS demonstrated that the difference between the two groups was not statistically significant (HR $1.02,95 \%$ CI $0.32-3.20, P=0.98$; Figure 3 ). A random effects model was used because significant heterogeneity was found between the trials $\left(I^{2}>50 \%\right)$.

\section{Subgroup analysis and sensitivity analysis}

There was significant heterogeneity among the studies $\left(I^{2}=63 \%\right)$, so we conducted subgroup meta-analysis to explore whether the heterogeneity was due to different antibody source, type, or methods. As shown in Table 2, a significantly superior OS was observed in the PD-L1-negative group when mouse antibodies were used as primary anti-PD-L1 antibodies (HR 1.85, 95\% CI 1.38-2.47, $P<0.0001)$. However, with respect to the rabbit antibodies, the difference in survival between the groups was not significant (HR 1.38, 95\% CI 0.66-2.90, $P=0.39$ ). Moreover, all PD-L1-negative patients presented significantly longer OS when using monoclonal antibodies (HR 1.87, 95\% CI 1.41-2.47, $P<0.0001)$. However, the use of a polyclonal antibody (HR 1.26, 95\% CI 0.54-2.94, $P=0.60$ ) indicated no significant difference in survival between the two trials. As for methods, when using the IHC method, the combined OS presented an adverse prognostic effect of PD-L1 expression (HR 1.63, 95\% CI 1.06-2.50, $P=0.02$ ). We were not able to evaluate the prognostic value of PD-L1 by the enzymelinked immunosorbent assay method in the subgroup analysis due to a lack of data.

\section{Publication bias}

A funnel plot of every two groups being compared was applied with HR as the $\mathrm{x}$-axis and standard error (SE) 


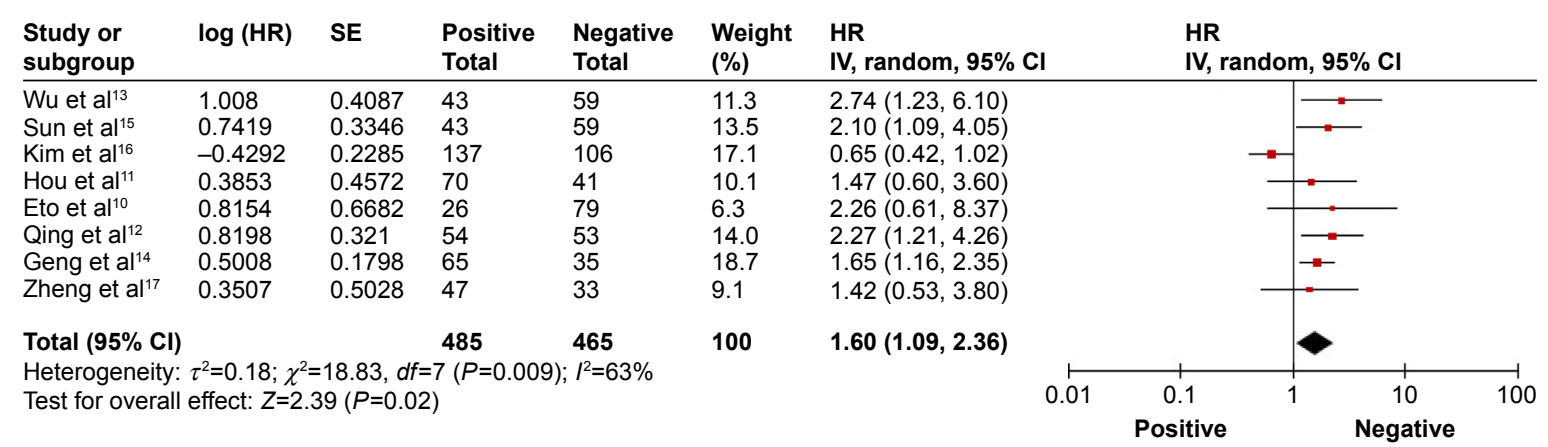

Figure 2 Overall response rate of programmed cell death-ligand I-positive versus-negative cases in patients with gastric cancer.

Notes: The summary HR and $95 \% \mathrm{Cls}$ are also shown (according to the random effect estimations). Weights are from random effects analysis.

Abbreviations: $\mathrm{Cl}$, confidence interval; HR, hazard ratio; SE, standard error; IV, inverse variance.

$(\log \mathrm{HR})$ as the y-axis. As shown in Figure 4, the plot was symmetric, suggesting that the publication bias was small.

\section{Discussion}

A number of studies demonstrated that PD-L1 plays a key role in cancer immune escape. ${ }^{20-22} \mathrm{~T}$-cell receptors of tumor-infiltrating lymphocytes recognize tumor-specific antigens when the antigens are presented by the major histocompatibility complex on cancer cells. Immune escape involves a loss of cancer antigens, a lack of $\mathrm{T}$ cells or antibodies, exhausted $\mathrm{T}$ cells, exhausted regulatory $\mathrm{T}$ cells, etc. Cancer cells can express PD-L1 to inhibit T cell-mediated antitumor immunity in response to a normal immune attack. $^{22,23}$ Alternatively, PD-L1 expression on tumor cells could lead to tumor cell immune evasion by inducing apoptosis of specific CD8+ cytolytic T cells. Some studies have demonstrated that the expression of PD-L1 on mouse P815 tumors increased the apoptosis of activated tumor-reactive $\mathrm{T}$ cells and promoted the growth of tumors in vivo. ${ }^{21}$

In our study, we have combined eight published studies with 950 patients for meta-analysis, which indicated that GC patients with positive expression of PD-L1 had significantly poorer OS than those with negative expression. However, PD-L1 overexpression was not associated with DFS of patients with GC. There are several possible explanations for this consequence. First, some studies have demonstrated that once PD-L1 is activated, cancer cells can evade immune response and continue to proliferate, and this fact can explain why patients with PD-L1 overexpression have poorer OS. ${ }^{21}$ Second, the numbers of the studies that reported DFS are too small and the relatively short period of DFS of patients with GC may affect our results.

We conducted subgroup analysis to explore the relationship between PD-L1 positivity and negativity for OS according to resources and the types of antibodies. We found that mouse antibodies and monoclonal antibodies were strongly correlated with the prognostic value of PD-L1. No heterogeneity was found in the subgroup analysis of either group $\left(I^{2}=0\right)$. However, there was not a statistically significant difference when using the rabbit antibodies $\left(I^{2}=59 \%\right)$ or the polyclonal antibodies $\left(I^{2}=61 \%\right)$ as the primary anti-PD-L1 antibody. However, controversial results were reported by relevant studies. ${ }^{10,11}$ The most likely reason is that antibodies cross-react with other proteins or that the numbers of the studies and patients included are relatively small. The pooled OS presented an unfavorable prognosis for patients with PD-L1 overexpression using the IHC method. Considering the consistent trends in all the subgroups with regard to methods and antibodies, a lack of uniform methods and criteria should not be a barrier to

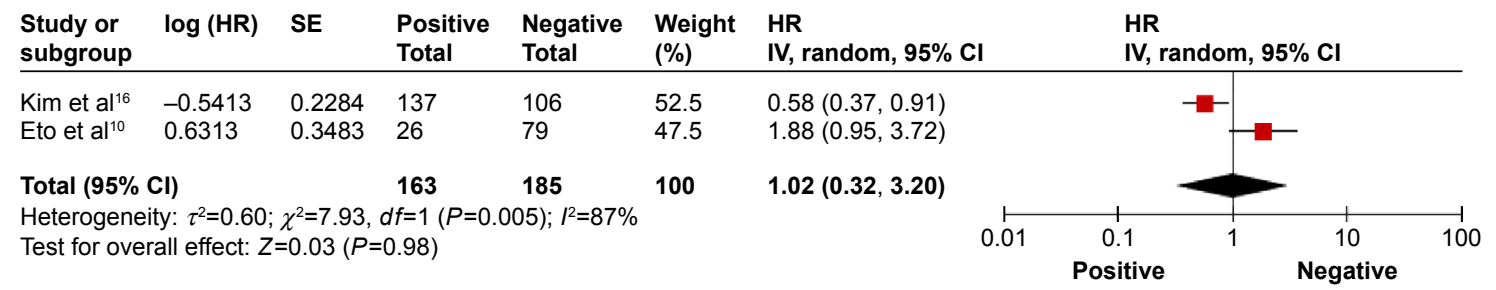

Figure 3 Progression-free survival of PD-LI-positive versus-negative cases in patients with gastric cancer.

Notes: The summary HR and $95 \% \mathrm{Cls}$ are also shown (according to the random effect estimations). Weights are from random-effects analysis.

Abbreviations: $\mathrm{Cl}$, confidence interval; HR, hazard ratio; PD-LI, programmed cell death-ligand I; SE, standard error; IV, inverse variance. 
Table 2 Summary of subgroup analysis results in studies that reported OS stratified by PD-LI status

\begin{tabular}{|c|c|c|c|c|c|c|c|}
\hline \multirow[t]{2}{*}{ Subgroup } & \multirow{2}{*}{$\begin{array}{l}\text { No of } \\
\text { studies }\end{array}$} & \multicolumn{2}{|c|}{ Heterogeneity } & \multirow{2}{*}{$\begin{array}{l}\text { Analysis } \\
\text { model }\end{array}$} & \multicolumn{2}{|c|}{ Effect size } & \multirow{2}{*}{$\begin{array}{l}\text { HR for OS } \\
(95 \% \mathrm{Cl})\end{array}$} \\
\hline & & $P$-value & $I^{2}(\%)$ & & $\mathbf{Z}$ & $P$-value & \\
\hline \multicolumn{8}{|c|}{ Antibody source } \\
\hline Mouse & 3 & 0.48 & 0 & Fixed effect & 4.16 & $<0.0001$ & $1.85(1.38,2.47)$ \\
\hline Rabbit & 4 & 0.008 & 75 & Random effect & 0.86 & 0.39 & $1.38(0.66,2.90)$ \\
\hline NA & 1 & & & & & & $1.42(0.53,3.80)$ \\
\hline \multicolumn{8}{|c|}{ Antibody type } \\
\hline Monoclonal & 4 & 0.67 & 0 & Fixed effect & 4.32 & $<0.0001$ & $1.87(\mathrm{I} .4 \mathrm{I}, 2.47)$ \\
\hline Polyclonal & 3 & 0.005 & 81 & Random effect & 0.53 & 0.60 & $1.26(0.54,2.94)$ \\
\hline NA & I & & & & & & I. $42(0.53,3.80)$ \\
\hline \multicolumn{8}{|l|}{ Methods } \\
\hline $\mathrm{IHC}$ & 7 & 0.004 & 68 & Random effect & 2.25 & 0.02 & \\
\hline ELISA & I & & & & & & I. $42(0.53,3.80)$ \\
\hline
\end{tabular}

Abbreviations: $\mathrm{Cl}$, confidence interval; ELISA, enzyme-linked immunosorbent assay; HR, hazard ratio; IHC, immunohistochemistry; NA, not available; OS, overall survival; PD-LI, programmed cell death-ligand I.

a pooled analysis to illustrate the prognostic significance of PD-L1 in GC.

We were the first to perform a meta-analysis on the association of the expression of PD-L1 and the prognosis of patients with GC. However, several limitations existed. 1) All the studies are based on an Asian population, including five from People's Republic of China, two from Japan, and one from Korea. There are significant differences, such as etiology, biology features, clinical types, and prognosis, in the risk of $\mathrm{GC}$ in different ethnic groups within a given geographical area. Due to a lack of statistics on the Western population, we cannot assess the prognosis of PD-L1 overexpression in Western patients. 2) Another potential source of bias is related to the method of $\mathrm{HR}$ and $95 \% \mathrm{CI}$ extrapolation. If these statistics were not reported by the authors, we calculated them from the data available or extrapolated them from the survival curves. It is possible that initial data errors occurred. In the future, large, well-designed prospective cohort studies with better

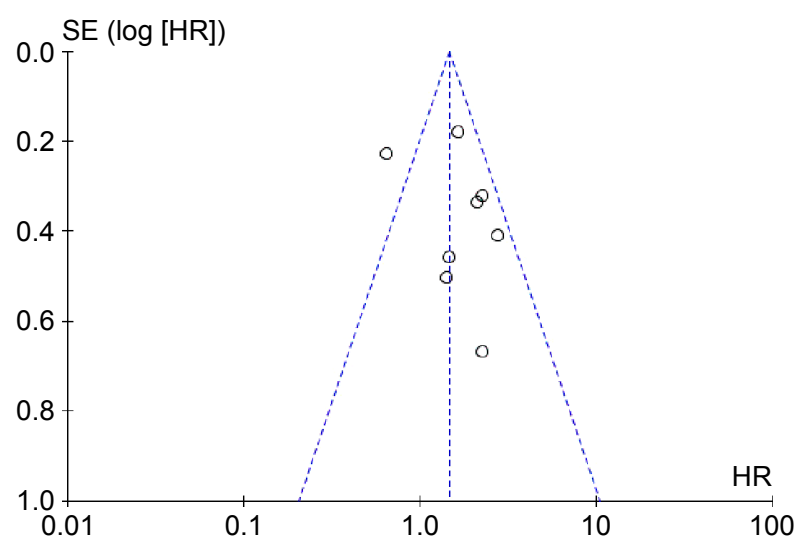

Figure 4 Funnel plot of the eight evaluable studies assessing programmed cell death-ligand I in gastric cancer.

Abbreviations: HR, hazard ratio; SE, standard error. exposure assessment are warranted to confirm the findings from our study and provide a higher level of evidence.

Regardless of the aforementioned limitations, our systematic review provides the best possible estimate of the correlation between the overexpression and clinical significance of PD-L1 in GC. Recently, several studies demonstrated that therapies targeting PD-L1 display clinical responses in patients with several cancer types, but the results for GC were unclear. ${ }^{8,9}$ Our results imply that further study is needed to clarify the different prognostic and therapeutic prediction values of PD-L1 expression for GC in tumor tissue and blood serum.

\section{Conclusion}

In conclusion, our results indicate that the evaluation of PD-L1 overexpression in GC tissue or blood serum may be useful in the future as a novel prognostic factor. Nevertheless, because the number of included trials was small, welldesigned, multicenter randomized controlled trials should be performed.

\section{Acknowledgment}

The authors thank the members of their laboratory for their helpful discussions.

\section{Disclosure}

The authors report no conflicts of interest in this work.

\section{References}

1. Torre LA, Bray F, Siegel RL, Ferlay J, Lortet-Tieulent J, Jemal A. Global cancer statistics, 2012. CA Cancer J Clin. 2015;65(2):87-108.

2. Fitzmaurice C, Dicker D, Pain A, et al. The global burden of cancer 2013. JAMA Oncol. 2015;1(4):505-527.

3. Chen W, Zheng R, Zeng H, Zhang S, He J. Annual report on status of cancer in China, 2011. Chin J Cancer Res. 2015;27(1):2-12.

4. Hanahan D, Weinberg RA. Hallmarks of cancer: the next generation. Cell. 2011;144(5):646-674. 
5. Schreiber RD, Old LJ, Smyth MJ. Cancer immunoediting: integrating immunity's roles in cancer suppression and promotion. Science. 2011; 331(6024):1565-1570.

6. Mittal D, Gubin MM, Schreiber RD, Smyth MJ. New insights into cancer immunoediting and its three component phases - elimination, equilibrium and escape. Curr Opin Immunol. 2014;27:16-25.

7. Merelli B, Massi D, Cattaneo L, Mandala M. Targeting the PD1/ PD-L1 axis in melanoma: biological rationale, clinical challenges and opportunities. Crit Rev Oncol Hematol. 2014;89(1):140-165.

8. Brahmer JR, Tykodi SS, Chow LQ, et al. Safety and activity of antiPD-L1 antibody in patients with advanced cancer. N Engl J Med. 2012; 366(26):2455-2465.

9. Taneja SS. Re: Safety and activity of anti-PD-L1 antibody in patients with advanced cancer. $J$ Urol. 2012;188(6):2148-2149.

10. Eto S, Yoshikawa K, Nishi M, et al. Programmed cell death protein 1 expression is an independent prognostic factor in gastric cancer after curative resection. Gastric Cancer. 2016;19(2):466-471.

11. Hou J, Yu Z, Xiang R, et al. Correlation between infiltration of FOXP3+ regulatory $\mathrm{T}$ cells and expression of B7-H1 in the tumor tissues of gastric cancer. Exp Mol Pathol. 2014;96(3):284-291.

12. Qing Y, Li Q, Ren T, et al. Upregulation of PD-L1 and APE1 is associated with tumorigenesis and poor prognosis of gastric cancer. Drug Des Devel Ther. 2015;9:901-909.

13. Wu C, Zhu Y, Jiang J, Zhao J, Zhang XG, Xu N. Immunohistochemical localization of programmed death-1 ligand-1 (PD-L1) in gastric carcinoma and its clinical significance. Acta Histochem. 2006;108(1):19-24.

14. Geng Y, Wang H, Lu C, et al. Expression of costimulatory molecules B7-H1, B7-H4 and Foxp3+ Tregs in gastric cancer and its clinical significance. Int J Clin Oncol. 2015;20(2):273-281.
15. Sun $\mathrm{J}, \mathrm{Xu} \mathrm{K}, \mathrm{Wu} \mathrm{C}$, et al. PD-L1 expression analysis in gastric carcinoma tissue and blocking of tumor-associated PD-L1 signaling by two functional monoclonal antibodies. Tissue Antigens. 2007;69(1): 19-27.

16. Kim JW, Nam KH, Ahn SH, et al. Prognostic implications of immunosuppressive protein expression in tumors as well as immune cell infiltration within the tumor microenvironment in gastric cancer. Gastric Cancer. 2016;19(1):42-52.

17. Zheng Z, Bu Z, Liu X, et al. Level of circulating PD-L1 expression in patients with advanced gastric cancer and its clinical implications. Chin J Cancer Res. 2014;26(1):104-111.

18. Tierney JF, Stewart LA, Ghersi D, Burdett S, Sydes MR. Practical methods for incorporating summary time-to-event data into meta-analysis. Trials. 2007;8:16

19. DerSimonian R, Laird N. Meta-analysis in clinical trials. Control Clin Trials. 1986;7(3):177-188.

20. Iwai Y, Ishida M, Tanaka Y, Okazaki T, Honjo T, Minato N. Involvement of PD-L1 on tumor cells in the escape from host immune system and tumor immunotherapy by PD-L1 blockade. Proc Natl Acad Sci US A. 2002;99(19):12293-12297.

21. Dong H, Strome SE, Salomao DR, et al. Tumor-associated B7-H1 promotes T-cell apoptosis: a potential mechanism of immune evasion. Nat Med. 2002;8(8):793-800.

22. Blank C, Kuball J, Voelk1 S, et al. Blockade of PD-L1 (B7-H1) augments human tumor-specific T cell responses in vitro. Int J Cancer. 2006; 119(2):317-327.

23. Lu J, Lee-Gabel L, Nadeau MC, Ferencz TM, Soefje SA. Clinical evaluation of compounds targeting PD-1/PD-L1 pathway for cancer immunotherapy. J Oncol Pharm Pract. 2015;21(6):451-467.
OncoTargets and Therapy

\section{Publish your work in this journal}

OncoTargets and Therapy is an international, peer-reviewed, open access journal focusing on the pathological basis of all cancers, potential targets for therapy and treatment protocols employed to improve the management of cancer patients. The journal also focuses on the impact of management programs and new therapeutic agents and protocols on

\section{Dovepress}

patient perspectives such as quality of life, adherence and satisfaction The manuscript management system is completely online and includes a very quick and fair peer-review system, which is all easy to use. Visit http://www.dovepress.com/testimonials.php to read real quotes from published authors. 